\title{
ERYTHEMA NODOSUM LEPROSUM- A CASE REPORT.
}

Pragnya Paramita Panda, P. R. Lenka, K. Panigrahi, D. Pattnaik

1. Assistant Professor, Department of Microbiology, KIMS, Bhubaneswar, Odisha.

2. Tutor, Department of Microbiology, KIMS, Bhubaneswar, Odisha.

3. Assistant Professor, Department of Microbiology, KIMS, Bhubaneswar, Odisha.

4. Professor, Department of Microbiology, KIMS, Bhubaneswar, Odisha.

\section{CORRESPONDING AUTHOR}

Dr. Pragnya Paramita Panda,

Asst. Professor, Dept. of Microbiology,

KIMS, Bhubaneswar, Odisha.

E-mail: bhavaarya@yahoo.com

Ph: 00917873762680.

ABSTRACT: Leprosy is an ancient disease that has survived into the modern ages despite an intense effort to eliminate it worldwide.[1] Here we report a case of recurrent, multiple ulcerative lesions with pitting edema of both lower extremities, in a 62 years old male. He was a diagnosed case of Hansen's disease with irregular treatment since 2 years. All biochemical parameters were within normal limits. Rheumatoid factor, ANA, ELISA for HIV and VDRL were negative. Modified ZN staining (using 5\% sulphuric acid) shows acid fast bacilli (grading +++), arranged in globi, $65 \%$ were fragmented \& $35 \%$ were uniformly stained. Organisms isolated in bacterial culture were Proteus mirabilis \& Staphylococcus aureus \& Candida spp isolated in fungal culture. Patient was admitted and was started on MDT-MB along with prednisolone.

KEY-WORDS: Erythema nodosum leprosum, Mycobacterium leprae, Modified ZN stain

INTRODUCTION: Leprosy is an ancient infectious disease caused by Mycobacterium leprae that affects the skin and peripheral nerves. A wide clinical spectrum of disease exists, from the tuberculoid pole [associated with a vigorous Th1 response, relatively few bacilli, and limited well-defined lesions] to the lepromatous pole [associated with aggressive Th2 response, many bacilli, and diffuse symmetric lesions].[2] Though it is a chronic disease, it has tendency for debilitating acute exacerbations termed reactions; Type 1 Reaction [Reversal Reaction] and Type 2 Reaction [Erythema Nodosum Leprosum (ENL)].[1] Erythema nodosum leprosum, is an acute inflammatory reaction seen in patients with lepromatous leprosy or occasionally in borderline lepromatous leprosy.[3] Though it is usually seen during the course of treatment it may occur in previously untreated patients as well.[4] The lesions are erythematous painful tender papules and nodules. In mild reaction nodules are small in number and spontaneously resolve leaving behind hyperpigmented macules. ${ }^{[5]}$ In severe reactions, nodules tend to increase in size and ulcerate. Vesiculobullous, pustular, ulcerated, and hemorrhagic and erythema multiforme-like lesions have been reported in ENL.[6] In this article, we have discussed a case which presented to us with recurrent, multiple ulcerative lesions with pitting edema of both lower extremities. The case was admitted in our hospital, Pradumna Bal Memorial Hospital, KIMS, Bhubaneswar, where he was investigated, diagnosed and started on treatment.

CASE REPORT: A 62 year old male was presented with multiple painful ulcerated skin lesions over both the lower extremities and gluteal areas of 3 months duration. He had history of recurrent attacks of papulo-pustular lesions, and ulcerations since two years. The skin lesion 


\section{CASE REPORT}

first started over the gluteal region and slowly developed over the other sites. Current episode was most severe one and associated with low grade and intermittent fever.

On examination, he had multiple subcutaneous, erythematous blanching nodules on both the lower extremities and gluteal areas. A few nodules were crusted with serosanguinous discharge [Fig.1]. There were no other patches, plaques or nodules in other part of the body. There was associated pitting edema \& loss of temperature sensation of lower extremities. Haematological investigations showed $\mathrm{Hb}-10 \mathrm{gm} \%$, TLC $-12000 / \mathrm{mm}^{3}$ with $70 \%$ of neutrophils, ESR showed $30 \mathrm{~mm}$ in the 1 st hour, FBS- $90 \mathrm{mg} \%$. Urine analysis and all other biochemical parameters were within normal limits. Rheumatoid factor, ANA, HBsAg, HIV and VDRL were negative. Bacterial culture of pus showed growth of Staphylococcus aureus and Proteus mirabilis and Candida spp isolated in fungal culture. Modified ZN staining (using 5\% sulphuric acid) of pus shows acid fast bacilli (grading +++), arranged in globi, 65\% were fragmented \& $35 \%$ were uniformly stained. Skin biopsy showed atrophic epidermis, few foamy macrophages with small aggregates of neutrophils in the dermis and oedematous dermal vessels.

The patient was diagnosed as a case of lepromatous leprosy with type 2 reaction [ENL]. Patient was started on MDT MB along with prednisolone $40 \mathrm{mg} /$ day (with the plan to be tapered and stopped over 3 months period). At subsequent follow-up, patient's condition was better with healing of all the lesions.

DISCUSSION: Leprosy is a unique disease with a peculiarly long incubation period, a wide spectrum of clinical presentations related to host immunity and acute exacerbations termed reactions. ${ }^{[1]}$

Type 2 reaction or ENL occurs in patients having high load of leprosy bacilli (50\% cases of lepromatous leprosy and $25 \%$ of borderline lepromatous leprosy).[3] It can occur in the early stages of treatment and even after completion of the treatment with MDT, commonly within first three years after the start of treatment. However, $10 \%$ cases have been reported even before starting treatment or as the first clinical manifestation of leprosy. ${ }^{[4]}$ It is a multisystem disorder involving skin, peripheral nerves, and sometimes, the internal organs. It presents in the skin as crops of painful or tender, erythematous, cutaneous and subcutaneous nodules concentrated on the extremities.[1] They appear preferentially on cooler parts of the skin (face and outer surface of limbs and less frequently on the trunk) and usually spare the warmer parts. Rarely they can break down and suppurate / necrose producing Erythema Nodosum Necroticans (ulcerative ENL). The condition is often accompanied by fever, neuritis, and inflammation of internal organs in varying degrees. Individual patients may have a single bout of ENL or chronic recurrent manifestations.

The pathogenesis of ENL involves immune complex deposition and dysfunction of cell mediated immunity. Activation of complement and migration of neutrophils with release of tissue damaging enzymes, including IL-12 and TNF- $\alpha$ are important factors in the evolution of the disease. Such patients have poor cellular immunity to M. leprae, abundant bacilli in cutaneous and peripheral nerves, and a strong polyclonal antibody response with high levels of circulating immunoglobulins..$^{[7]}$

As immune complex deposition \& defective CMI are the main cause in pathogenesis of ENL, immunosuppressants and TNF- $\alpha$ inhibitors have a role in treatment. Thalidomide and corticosteroids are the mainstays of treatment. Various other modalities of treatment are clofazimine, azathioprine, cyclosporine, methotrexate, antimony compound..[7] Newer modalities 
of treatment are IV Immunoglobulins, and the new non-steroidal anti-inflammatory drug, Tenidap. ${ }^{[8]}$

Our case presents with multiple, painful ulcerated skin lesions over both the lower extremities and gluteal areas since 3 months and was on irregular treatment MB leprosy since 2 years. The case gets complicated by secondary bacterial and fungal infections due to delay in diagnosis. The case was interesting because ENL occurring after treatment with MDT-MB and presenting with extensive cutaneous ulceration is a very rare entity. But the diagnosis can be made on the basis of simple microscopy \& culture which helps in patient's recovery by proper treatment and follow up.

So occurrence of tender, red, ulcerative nodules in leprosy patients on treatment should always prompt the treating physician to rule out ENL by simple clinical and laboratory tests. Early diagnosis \& definitive treatment with glucocorticoid is essential to prevent secondary complications

\section{REFERENCES:}

1. Smitha Prabhu, S D Shenoi, Sathish Pai B, Sripathi H. Erythema nodosum leprosum as the presenting feature in multibacillary leprosy: Dermatology Online Journal 15(6):15.

2. Boggild AK, Keystone JS, Kain KC. Leprosy: a primer for Canadian physicians. CMAJ 2004;170:71-8.

3. 3.. Pocaterra L, Jain S, Reddy R, Muzaffarullah S et al. Clinical course of Erythema nodosum leprosum: an 11-year cohort study in Hyderabad, India. Am J Trop Med Hyg 2006;74:868-79.

4. Rea TH and Levan NE. Erythema nodosum leprosum in a general hospital. Arch Dermatol 1975;111:1575-80.

5. Hosahalli Rajaiah Yogeesh HR, Sujatha Chankramath, Hari Kishan Kumar Yadalla, Shameem Shariff et al.Type 2 lepra reaction (enl) presenting with extensive cutaneous ulcerations: Our Dermatol Online. 2012; 3(1): 17-20.

6. Meyerson MS: Erythema nodosum leprosum. Int J Dermatol 1996; 35: 389-392.

7. Scollard DM, Adams LB, Gillis TP, Krahenbuhl JL, et al. The continuing challenges of leprosy. Clin Microbiol Rev 2006;19:338-81.

8. van Veen NHJ, Lockwood DNJ, van Brakel WH, Ramirez Jr J, Richardus JH. Interventions for erythema nodosum leprosum. (Protocol) Cochrane Database of Systematic Reviews 2008, Issue 1. Art. No.: CD006949. DOI: 10.1002/14651858.CD006949. 
Fig.1: Multiple subcutaneous, erythematous blanching nodules on both the lower extremities.

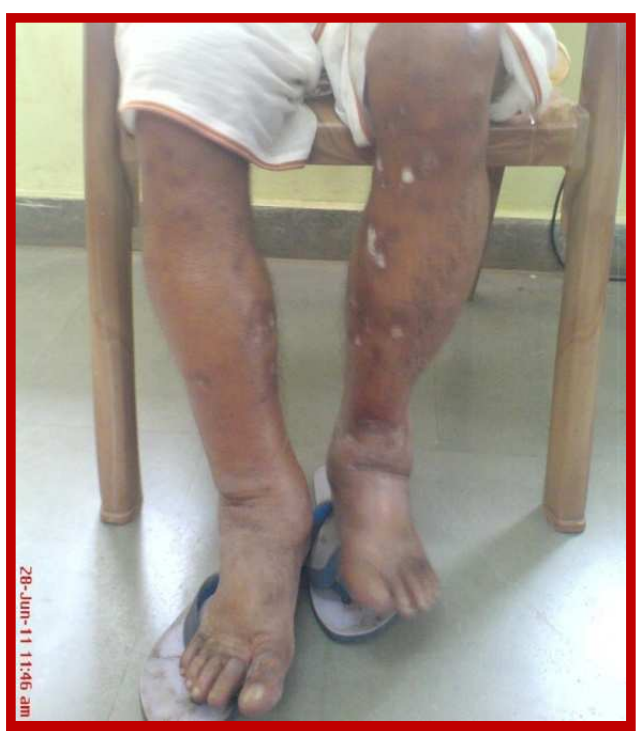

